\title{
In vitro effects of dextran sulfate sodium on a Caco-2 cell line and plausible mechanisms for dextran sulfate sodium-induced colitis
}

\author{
YOSHIO ARAKI, HIROYUKI SUGIHARA and TAKANORI HATTORI
}

First Department of Pathology, Shiga University of Medical Science, Seta Tsukinowa, Otsu 520-2192, Shiga, Japan

Received May 29, 2006; Accepted August 8, 2006

\begin{abstract}
Pathogenic mechanisms responsible for inflammatory bowel disease (IBD) are poorly understood. In an IBD animal model, the oral administration of polysaccharides such as dextran sulfate sodium (DSS) induces colitis, which exhibit several clinical and histological features for IBD. However, pathogenic factors in the development of colitis remain unclear. Therefore, we investigated possible mechanisms for DSS-induced colitis, and mainly focused on biological responses from an intestinal epithelial cell line, Caco-2. Cytotoxicity and cytokine release were measured using MTS assays and ELISA, respectively. The effect of DSS on the transepithelial electrical resistance (TEER) of Caco-2 cell monolayers was also evaluated. Cell cycle progression was estimated using antibodies directed against p53 and cdc-2 proteins. The generation of reactive oxygen species (ROS) was measured using a DCFH-DA method. PyridylaminoDSS (PA-DSS) was used as a fluorometric label in order to investigate fluorescence-microscopically the location of DSS in Caco- 2 cells. DSS induced cytotoxicity on Caco- 2 cells at $5 \%$. DSS also induced strong TEER decrease at $3 \%$. DSS induced the weak release of IL- 8 , IL-6, and TGF- $B 1$. Remarkably DSS arrested Caco- 2 cell cycle and reduced the intracellular generation of ROS. Under fluorescence microscopy, PA-DSS entered cells and bound to the nucleus, indicating this binding of DSS may be involved in the cell cycle arrest of Caco-2 cells. The cell cycle arrest and reduced intracellular generation of ROS may be involved during initiation or throughout the early stages of DSS-induced colitis.
\end{abstract}

\section{Introduction}

Human inflammatory bowel disease (IBD), including Crohn's disease (CD) and ulcerative colitis (UC), is a chronic, relapsing, and remitting condition that exhibits various

Correspondence to: Dr Yoshio Araki, 6-13-20 Aoyama, Otsu, Shiga 520-2101, Japan

E-mail: bileacidaraki@hotmail.com

Key words: dextran sulfate sodium, colitis, cell cycle, reactive oxygen species features of immunological inflammation. IBD is characterized by an infiltration of large numbers of neutrophils, monocytes, and lymphocytes in the large and/or small intestine, diarrhea, bloody stools, abdominal pain, body weight loss, anemia, and leukocytosis. IBD affects at least 1 in 1000 people in Western countries (1). Despite many investigations, the etiology and pathogenic mechanisms responsible for IBD remain poorly understood. Experimental animal models of IBD are being developed to investigate pathogenesis and improve treatment options. Most commonly, colitis is induced by administering polysaccharides such as dextran sulfate sodium (DSS) or carrageenan. DSS is a heparin-like polysaccharide containing $\sim 17 \%$ sulfur with up to three sulfates per glucose molecule (2). It has been reported that DSS-induced colitis exhibits some clinical and histological features of UC. For example, similar to UC patients, DSS initially induces colonic lesions in the distal colon, which then spread to the whole colon (3). In this model, the development of colitis is dependent on the molecular weight and sulfation of DSS, in addition to dosage and duration of administration (4). With respect to pathogenic factors in the development of DSS-induced colitis, previous reports have postulated the importance of various factors such as local immunological disturbances (5), the activation of mucosal macrophages (6), obliteration of the crypt lumina (7), and changes in intestinal microflora (8). Recently, it has been suggested that reactive oxygen species may play an important role in the intestinal inflammation (9). However, precise mechanisms responsible for DSS-induced colitis remain unclear. The interaction between DSS and the intestinal epithelium also remains unclear, especially in earlier stages of the disease, when DSS initially contacts intestinal epithelia.

Therefore, we tried to investigate possible mechanisms for DSS-induced colitis. We performed this study using the Caco-2 intestinal cell line focusing on DSS-induced cytotoxicity, barrier breakage, the release of biological substances from cells, cell cycle alterations and generation of ROS. Caco-2 biological responses may be relevant to inflammatory reactions, especially in the earlier stages of colitis.

\section{Materials and methods}

Caco-2 preparation. The human colon cancer-derived Caco-2 intestinal epithelial cell system has been widely used as an in vitro model of the intestinal epithelium (10). When confluent and allowed to mature on permeable inserts, Caco- 2 cells 
form tight junctions and attain many morphological and functional characteristics of enterocytes (11). In this study, passages 15-21 were used. Caco-2 cells were cultured in Dulbecco's modified Eagle's minimum essential medium (DMEM, pH 7.4) supplemented with $25 \mathrm{mM}$ glucose, $10 \%$ inactivated fetal bovine serum (FBS), $1 \%$ penicillinstreptomycin and $1 \%$ non-essential amino acid solution. Cells were maintained at $37^{\circ} \mathrm{C}$ in a humidified $5 \% \mathrm{CO}_{2}$ atmosphere.

Cytotoxicity (viability) tests. We used 5000 Da (Wako Pure Chemical Industries Ltd., Osaka, Japan) or 500000 Da (Nacalai Tesque Inc., Kyoto, Japan) DSS. Cell viability assays were performed according to our previously reported method (12). Briefly, cells were inoculated in 96-multiwell plates (Costar, Corning, NY, USA) at a cell density of $1.2 \times 10^{5}$ cells/well. After cells reached confluence, cells were incubated with serially diluted DSS for pre-determined time periods. DSS was dissolved in culture media and filter-sterilized using a $0.45 \mu \mathrm{m}$ filter. Viability was assayed by a commercially available kit (Cell Titer 96 ${ }^{\mathrm{TM}}$ AQueous, Promega, Madison, USA), which depends on the physiologic reduction of MTS to formazan. Analyses were performed in triplicate.

Determinations of transepithelial electrical resistance (TEER). We used both 5000 and 500000 Da DSS. For growth on porous filters, Caco-2 cells were grown in the same medium and plated at $3 \times 10^{5}$ cells $/ 100 \mu 1$ on Millicell-HA (Millipore, Bedford, MA), respectively. Cellular TEERs were measured with an electrical resistance system, Millicell-ERS (Millipore) according to our previous methods (13). Cells with stable TEER readings $>500 \Omega \mathrm{cm}^{2}$ were used (4-5 weeks post plating). DSS were exposed to apical sides of Caco-2 cell monolayers. Analyses were performed in triplicate.

Biochemical assays. Caco-2 cells were incubated in 24-well plates (Costar). After cells reached confluence, serial dilutions of 5000 Da DSS were exposed to cells for $10 \mathrm{~h}$. Supernatants were then collected, centrifuged at $14000 \mathrm{rpm}$ for $20 \mathrm{~min}$ to remove cellular debris, and stored at $-80^{\circ} \mathrm{C}$ until further use. Assay kits for IL-6, IL-8 and IL-10 levels in supernatants were purchased from BioSource Europe S.A. (Belgium), for TGF- $\beta 1$ and MMP-1 from Amersham Biosciences (UK), urokinase-type plasminogen activator (u-PA) from Chemicon (CA, USA), for bradykinin from Peninsula Laboratories, Inc. (CA, USA). Analyses were performed in triplicate. Some studies have indicated certain types of colitis such as DSSinduced colitis and human IBD, especially UC, may be induced through the production of hydrogen sulfide $\left(\mathrm{H}_{2} \mathrm{~S}\right)$, which exerts cytotoxic effects, in the gut lumen $(14,15)$. In addition, there is a possibility that cells can produce $\mathrm{H}_{2} \mathrm{~S}$ or sulfate from DSS. Therefore, we measured the production of $\mathrm{H}_{2} \mathrm{~S}$ or sulfate in Caco-2 cells following DSS exposure. Caco-2 cells were incubated in tissue culture flasks $\left(75 \mathrm{~cm}^{2}\right)$ (TPP, Switzerland). After Caco-2 cells reached confluence, $3 \%$ DSS was added to flasks and cells were incubated for $10 \mathrm{~h}$. Supernatants and cells were separately collected and analyzed. $\mathrm{H}_{2} \mathrm{~S}$ concentrations were analyzed according to a previously reported method using methylene blue (16). Sulfate concentrations were analyzed by HPLC using an IonPacAG4-SC

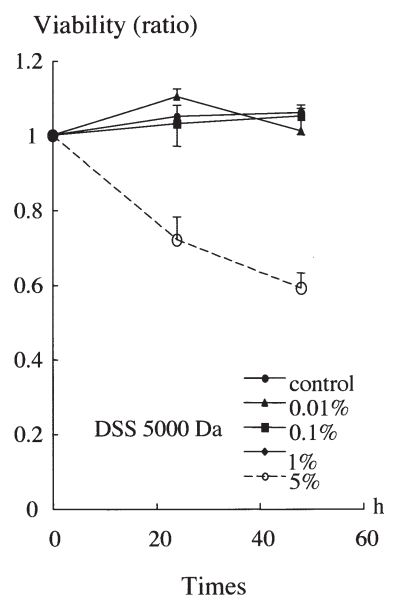

A

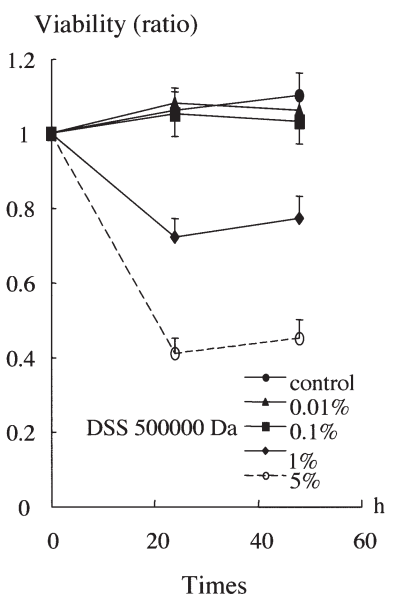

B
Figure 1. Cytotoxicities of DSS on Caco-2 cells. Relative numbers of viable cells were determined using an MTS assay (Materials and methods). Percent survival is expressed as the percentage of growth compared to corresponding untreated control cells. A and B show the percent survival at various concentrations of DSS at MW 5000 and 500000 Da, respectively. Each point represents the mean of three determinations.

column (4x50 mm) (Dionex Corporation, CA), an electric conductivity detector, and $2 \mathrm{mM}$ sodium carbonate $/ 1 \mathrm{mM}$ sodium hydrogen carbonate as an eluent.

Cell cycle assays. Caco-2 cells were incubated in chamber slides (Lab-Tek ${ }^{\circledR}$ Chamber slide system, Nalge Nunc International, Naperville, USA). After cells reached confluence, $1 \% 5000$ Da DSS was added to each chamber and cells were incubated. Primary antibodies against phospho-cdc2 (Tyr15) or phospho-p53 (Ser15) protein (Cell cycle/checkpoint sampler kit, Cell Signaling Technology, Inc., MA, USA), and ABC biotin/avidin were used to immunohistochemically stain cells.

The determination of intracellular reactive oxygen species (ROS) generation in Caco-2 cells. Intracellular ROS generation was measured according to our previously reported method using 2', 7'-dichlorodihydrofluorescein diacetate (DCFH-DA) (13). Briefly, DCFH-DA was dissolved in $\mathrm{Me}_{2} \mathrm{SO}$ and diluted with PBS to a final concentration of $100 \mu \mathrm{M}$. Cells were plated on a 48-well culture plate. After their confluent, medium was replaced by culture medium containing $2.5 \%$ for $1 \mathrm{~h}$. Then, cells were treated with indicated 5000 Da DSS for $1 \mathrm{~h}$. The medium was then removed and loaded with $100 \mu \mathrm{M}$ DCFHDA for $30 \mathrm{~min}$ at $37^{\circ} \mathrm{C}$. The loading was terminated by washing the cells with PBS. A microplate fluorescence reader (MTP-600F, Hitachi, Tokyo) was set to an excitation wavelength of $490 \mathrm{~nm}$ and an emission wavelength of $530 \mathrm{~nm}$ and used for DVF detection. The increase in fluorescence for each treatment was calculated by the relative fluorescence of each treatment compared to a control consisting of untreated cells.

The location of DSS in Caco-2 cells. Pyridylamino-DSS (PADSS) was prepared according to our novel methods (17). Caco-2 cells were incubated in chamber slides. After cells reached confluence, $1 \% 5000$ Da PA-DSS was added to each 


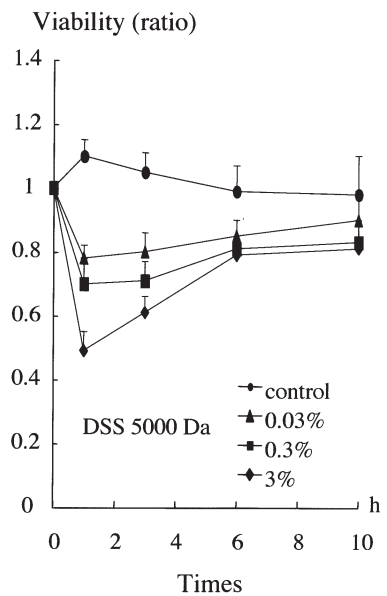

A

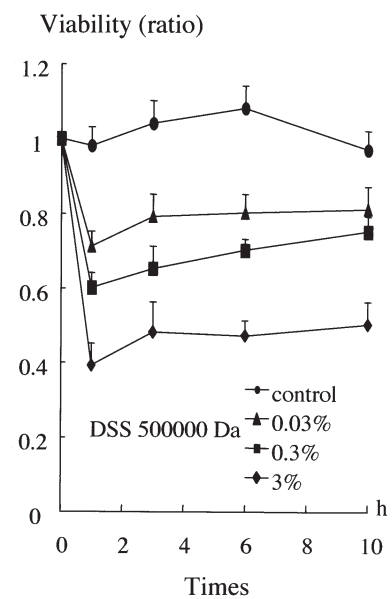

B

Figure 2. DSS effects on Caco-2 cell TEERs. Plotted is the percentage of initial resistance $\left(\sim 500 \Omega \mathrm{cm}^{2}\right)$ versus time. A and B show the TEER decreases at various concentrations of DSS at MW 5000 and 500000 Da, respectively. Values are expressed as the percentage of TEER compared to corresponding control cells without DSS treatment. Each point represents the mean of three determinations.

IL-8

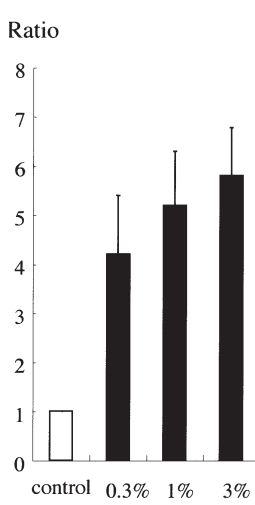

A

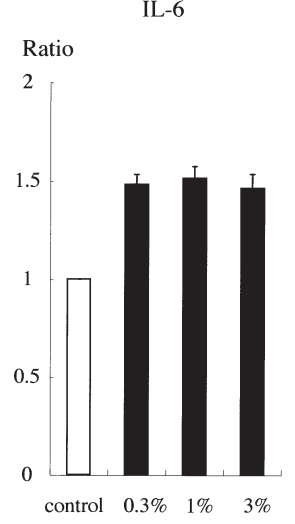

B

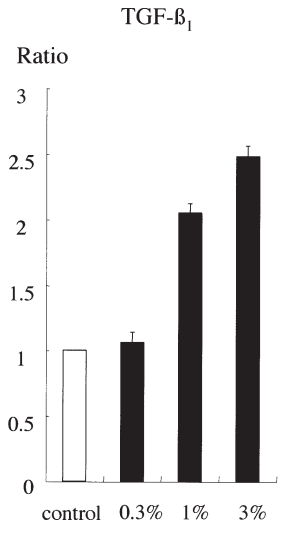

$\mathrm{C}$

Figure 3. IL-8, IL-6 and TGF- 31 released into culture media by DSS-treated Caco-2 cells. Caco-2 cells were exposed to 5000 Da DSS at the indicated concentrations for $10 \mathrm{~h}$. Supernatants were then collected and IL-8 (A), IL-6 (B) and TGF-B1 (C) levels were measured. Values are expressed as percentages of levels compared to corresponding untreated control cells. Results represent the mean of three determinations.

chamber and cells were incubated. After $6 \mathrm{~h}$ of incubation, cells were wash 3 times by PBS and fixed with $20 \%$ glutaraldehyde for $20 \mathrm{~min}$, washed in PBS, and then mounted with PBS:glycerol (3:1, vol/vol). The fluorescence was visualized under a fluorescence microscope (Labophot-2, Nikon, Tokyo, Japan).

Statistics. The data were expressed as means \pm SEM. The significance of differences between the means was determined by a one-way analysis of variance, followed by Fisher's protected least significant difference test. P-values $<0.05$ were considered to be statistically significant.

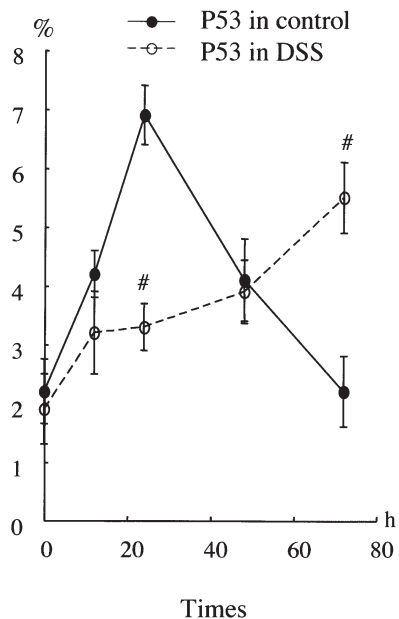

A

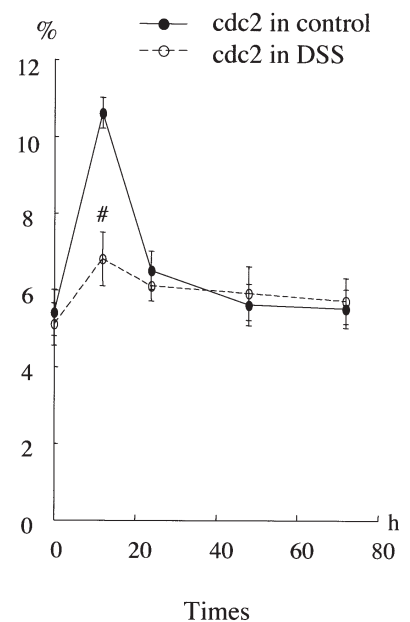

B
Figure 4. Cell cycle analysis in Caco-2 cells following DSS exposure. Caco-2 cells were exposed to $1 \% 5000$ Da DSS for the times indicated. Primary antibodies directed against phospho-cdc2 (Tyr15) or phospho-p53 (Ser15) and $\mathrm{ABC}$ biotin/avidin were used to immunohistochemically stain cells. Immunohistochemically-positive cells were counted. Each point represents the mean of three determinations. $\mathrm{P}<0.05$ vs. control.

\section{Results}

Cytotoxicity tests. Fig. 1 shows Caco-2 survival curves for cells exposed to 5000 and 500000 Da DSS. Survival curves of cells exposed to $5000 \mathrm{Da}$ at $0.01-1 \%$ were comparable to those of controls, but cytotoxic effects became clear at $5 \%$. As for 500000 Da DSS, cytotoxic effects were clear at $1 \%$. Cytotoxicity in 500000 Da DSS tended to be a bit stronger than that in $5000 \mathrm{Da}$ DSS.

Determination of TEERs. In both 5000 and 500000 Da DSS, the TEER of Caco-2 cell monolayers clearly decreased at $3 \%$. The TEER decrease in 500000 Da DSS tended to be a bit stronger than that in 5000 Da DSS (Fig. 2). These results might suggest that high concentrations of DSS in the gut lumen exert cytotoxicity and per se disrupt barrier functions of intestinal epithelia. On the other hand, we had to consider that these effects might be due to the high osmotic pressure of the DSS solution. In fact, the osmotic pressure of DSSmedium solutions increased to $3.81 \%$ and $1.74 \%$ as DSS concentrations increased to $1 \%$, respectively (data not shown). Therefore, we tested the cytoxicity and TEER decrease following a correction for increased osmotic pressure. The results, however, were the same as those of increased osmotic pressure. Therefore, we concluded that the osmotic pressures did not involve cytotoxicity and a TEER decrease (data not shown).

Biochemical assays. DSS (5000 Da) induced the weak release of IL-8, IL-6 and TGF-31 from Caco-2 cells (Fig. 3). On the other hand, 5000 Da DSS did not significantly induce the release of IL-10, MMP-1, u-PA, or bradykinin from Caco-2 cells (data not shown). In addition, the generation of $\mathrm{H}_{2} \mathrm{~S}$ or sulfate was not observed in supernatants or cells (data not shown). 


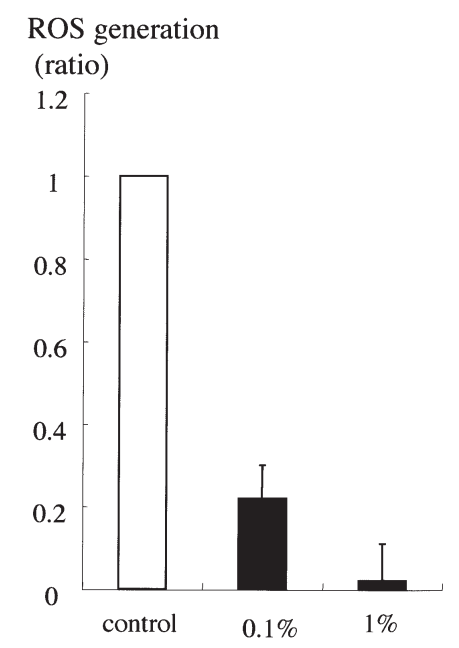

Figure 5. DSS prevents intracellular ROS generation in Caco-2 cells. Plotted is the ratio of the control value after $5 \mathrm{~h}$ versus time. DSS (5000 Da) inhibitory effects were observed at indicated concentrations on intracellular ROS generation. Values are expressed as percentages of levels compared to corresponding control untreated cells. Results represent the mean of three determinations.

Cell cycle assays. We investigated 5000 Da DSS effects on Caco-2 cell cycles using an immunohistochemical method (Fig. 4). In culture media, after cells reached confluence, the ratio of cells representing $\mathrm{p} 53$ protein, which is predominately expressed in $\mathrm{S}$ phase cells, was $\sim 2 \%$. After the culture medium was replaced, the ratio of cells representing p53 protein increased $\sim 7 \%$ at $24 \mathrm{~h}$, followed by a decrease, and reached $\sim 2 \%$ at $60 \mathrm{~h}$. In samples treated with $1 \% 5000$ Da DSS, the ratio of cells representing $\mathrm{p} 53$ protein gradually increased from $\sim 2 \%$ and maximally reached $\sim 5.5 \%$ at $60 \mathrm{~h}$. As for cdc 2 protein, which is predominately expressed in $\mathrm{M}$ phase cells, after cells reached confluence, the ratio of cells representing cdc2 protein was $\sim 3 \%$ in culture medium. After the culture medium was replaced, the ratio of cells representing cdc2 protein increased $\sim 11 \%$ at $12 \mathrm{~h}$, followed with a decrease, and reached $\sim 6 \%$ at $24 \mathrm{~h}$. DSS ( $1 \% 5000 \mathrm{Da})$ reduced the ratio of cells representing cdc 2 protein to $\sim 7 \%$ maximally.

ROS generation in Caco-2 cells. In culture medium, Caco-2 cells generated intracellular ROS in a time-dependent manner. DSS (5000 Da) generated less ROS in Caco- 2 cells at $5 \mathrm{~h}$ in a dose-dependent manner (Fig. 5). These concentrations actually induced no cytotoxic effects on cells.

The location of DSS in Caco-2 cells. Under a fluorescence microscope, PA-DSS entered cells and bound mainly the nucleus. Among the surface of nucleus, several more densely bound areas were recognized (Fig. 6).

\section{Discussion}

In this in vitro study, we observed DSS cytotoxic effects on Caco-2 cells at high concentrations $(5 \%$ in 5000 Da and $1 \%$ in $500000 \mathrm{Da}$, respectively). We previously observed that after an oral administration of 5000 Da DSS, the actual concentration of 5000 Da DSS in feces was compatible to that of

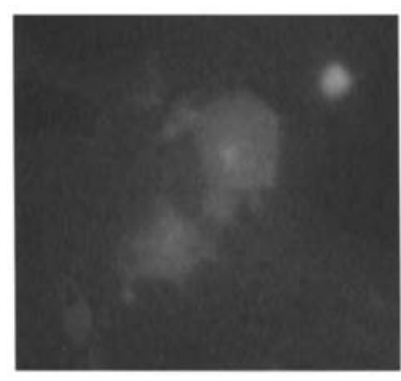

A

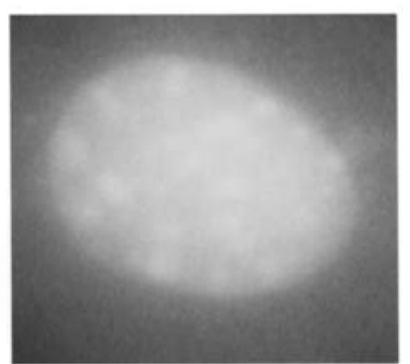

B
Figure 6. The location of Pyridylamino-DSS (PA-DSS) in Caco-2 cells. Caco-2 cells were incubated in chamber slides. After cells reached confluence, $1 \%$ PA-DSS (5000 Da) was added and the cells were incubated. After $6 \mathrm{~h}$, cells were wash and fixed, and then mounted with glycerol. The fluorescence was visualized under a fluorescence microscope (x200 magnification). (A) Control and (B) PA-DSS treated cells.

oral uptake in rats (17). That is, when rats are fed a diet containing 3\% $5000 \mathrm{Da}$ DSS, the concentration in feces reaches almost $3 \%$. In addition, low concentrations of DSS were unable to induce colitis (4). That is, $3 \%$ DSS is essential for induction of colitis. When we consider other cytotoxic substances in the environment exert their effects at nM-mM orders, DSS per se has weak cytotoxicity. As for the epithelial permeability of Caco-2 cells, previous in vivo studies also reported that oral DSS administration induced increased colonic mucosal permeability $(18,19)$. In this in vitro study, DSS also decreased the TEER of Caco- 2 cells. In particular, $3 \%$ DSS at both 5000 and 500000 Da exhibited a strong TEER decrease. Therefore, our TEER measurement data are also compatible to data from in vivo studies. In addition, these effects were not derived from increased osmorality of DSS solution.

As for biological responses, some cytokine profiles such as IL-6 in colonic mucosa were investigated in DSS-induced colitis (20). In this in vitro study, we evaluated the release of several cytokines and biological mediators from Caco- 2 cells. In particular, DSS influences in vitro fibrinogenic-fibrolytic systems such as B-FGF (21), collagen (22), procollagen (23), and bradykinin (24), or in vivo systems such as MMPs (25). As a result, DSS weakly induces the release of IL-8, IL-6 and TGF-ß1, and does not influence IL-10, MMP-1, u-PA, or bradykinin. Therefore, it may be that early stages of DSSinduced colitis involve cytotoxic effects to the epithelium cells rather than immunological or fibrolytic mechanisms. In addition, immunological or fibrolytic mechanisms may be involved in the following stage, where DSS reach to subepithelium space.

On the other hand, some studies have indicated that certain kinds of colitis such as DSS-induced colitis and human IBD, especially UC, may be induced through the production of $\mathrm{H}_{2} \mathrm{~S}$ in the gut lumen, which is supposed to be cytotoxic $(14,15)$. Therefore, we evaluated whether cells degrade DSS or whether $\mathrm{H}_{2} \mathrm{~S}$ or sulfate is produced from DSS. As a result, we did not observe $\mathrm{H}_{2} \mathrm{~S}$ or sulfate production in Caco-2 cells. At least 
epithelial cells do not have the capacity to produce $\mathrm{H}_{2} \mathrm{~S}$ or sulfate from DSS sulfurs. In other words, any $\mathrm{H}_{2} \mathrm{~S}$ in the gut lumen in DSS-colitis may derive from intestinal microflora rather than from the metabolism of intestinal epithelia.

The following two results were most remarkable. Firstly, DSS has been implicated in cell cycle arrest $(26,27)$, especially in G1 (28). Carrageenan, which belongs to the same sulfated polysaccharides as DSS also arrested the cell cycle (29). In addition, DSS inhibited DNA topoisomerases I and II (30). On the other hand, DSS controlled cell proliferation via DNA polymerase $\alpha$ (31). These reports were all in vitro studies. However, it has been suggested that the cell cycle increased in DSS-induced colitis in vivo (32) and clinical UC patients $(33,34)$. In this in vitro study, it is clear that DSS arrests cell cycles of Caco- 2 cells. Therefore, we consider the cell cycle promotion in DSS-induced colitis and UC patients as a following reaction on repair process from colitis.

Secondly, for the first time it has become clear that DSS strongly inhibits ROS generation in Caco- 2 cells at $0.1 \%$ of concentrations which do not exert cytotoxicity. DSS was not reported as a radical scavenger per se (35). Many previous in vivo studies reported increased mucosal generation of ROS in DSS-induced colitis $(9,36)$. Taken together, these results suggest that generated ROS in the inflammatory mucosa may be derived from inflammatory cells beneath the epithelium. Why the cell cycle was arrested or the intracellular ROS generation was inhibited remains unclear. We observed that PA-DSS entered into the cells and bound to the nucleus. However, it is still unclear whether this binding of DSS is involved in the cell cycle arrests. The intestinal epithelium cells have a frequent cell cycle, therefore cell cycle arrest may lead to breaks in the barrier and absorb functions. In addition, the reduction in ROS might represent the fact that DSS alters the intracellular energy metabolism because ROS are generated where energy metabolism exists. The early stage of DSS-induced colitis might involve cytostatic mechanisms, in other words, as if cells were in hibernation.

In conclusion, DSS arrested the cell cycle and strongly inhibited intracellular ROS generation in Caco- 2 cells. It is possible that these effects are involved in mechanisms of DSS-induced colitis, especially in the early stage of colitis.

\section{References}

1. Fiocchi C: Inflammatory bowel disease: etiology and pathogenesis. Gastroenterology 115: 182-205, 1998.

2. Ricketts CR: Dextran sulphate- $\alpha$ synthetic analogue of heparin. Biochem J 51: 129-133, 1952.

3. Araki Y, Andoh A, Fujiyama Y and Bamba T: Development of dextran sulphate sodium-induced experimental colitis is suppressed in genetically mast cell-deficient $W s / W s$ rats. Clin Exp Immunol 119: 264-269, 2000.

4. Okayasu I, Hatakeyama S, Yamada M, Ohkusa T, Inagaki Y and Nakaya R: A novel method in the induction of reliable experimental acute and chronic ulcerative colitis in mice. Gastroenterology 98: 694-702, 1990.

5. Kobayashi K, Asakura H, Hamada Y, Hibi T, Watanabe M, Yoshida T, Watanabe N, Miura S, Aiso S and Tsuchiya M: T lymphocyte subpopulations and immunoglobulin-containing cells in the colonic mucosa of ulcerative colitis; a morphometric and immunohistochemical study. J Clin Lab Immunol 25: 63-68, 1988.

6. Abraham R, Fabian RJ, Golberg L and Coulston F: Role of lysosomes in carrageenan-induced cecal ulceration. Gastroenterology 67: 1169-1181, 1974.
7. Iwanaga T, Hoshi O, Han $\mathrm{H}$ and Fujita T: Morphological analysis of acute ulcerative colitis experimentally induced by dextran sulfate sodium in the guinea pig: some possible mechanisms of cecal ulceration. J Gastroenterol 29: 430-438, 1994.

8. Onderdonk AB, Hermos JA and Bartlett JG: The role of the intestinal microflora in experimental colitis. Am J Clin Nutr 30: 1819-1825, 1977.

9. Araki Y, Andoh A and Fujiyama Y: The free radical scavenger edaravone suppresses experimental dextran sulfate sodiuminduced colitis in rats. Int J Mol Med 12: 125-129, 2003.

10. Quaroni A, Wands J, Trelstad RL and Isselbacher KJ: Epithelial cell culture from rat small intestine. J Cell Biol 80: 248-265, 1979.

11. Hidalgo IJ, Raub TJ and Borchardt RT: Characterization of human colon carcinoma cell line (Caco-2) as a model system of intestinal epithelial permeability. Gastroenterology 96: 736-749, 1989.

12. Araki Y, Fujiyama Y, Andoh A, Nakamura F, Shimada M, Takaya $\mathrm{H}$ and Bamba T: Hydrophilic and hydrophobic bile acids exhibit different cytotoxicities through cytolysis, interleukin-8 synthesis and apoptosis in the intestinal epithelial cell lines, IEC-6 and Caco-2 cells. Scand J Gastroenterol 36: 533-539, 2001.

13. Araki Y, Katoh T, Ogawa A, Bamba S, Andoh A, Koyama S, Fujiyama Y and Bamba T: Bile acid modulates transepithelial permeability via the generation of reactive oxygen species in the Caco-2 cell line. Free Radical Bio Med 39: 769-780, 2005

14. Furne JK, Suarez FL, Ewing SL, Springfield J and Levitt MD: Binding of hydrogen sulfide by bismuth does not prevent dextran sulfate-induced colitis in rats. Dig Dis Sci 45: 1439-1443, 2000.

15. Jorgensen J and Mortensen PB: Hydrogen sulfide and colonic epithelial metabolism: implications for ulcerative colitis. Dig Dis Sci 46: 1722-1732, 2001

16. Strocchi A, Furne JK and Levitt MD: A modification of the methylene blue method to measure bacterial sulfide production in feces. J Microbiol Methods 15: 75-82, 1992.

17. Araki Y, Andoh A, Fujiyama Y, Hata K, Makino J, Okuno T, Nakanura F and Bamba T: Application of 2-aminopyridine fluorescence labeling in the analysis of in vivo and in vitro metabolism of dextran sulfate sodium by size-exclusion highperformance liquid chromatography. J Chromatogr B Biomed Appl 753: 209-215, 2001

18. Kitajima S, Takuma S and Morimoto M: Changes in colonic mucosal permeability in mouse colitis induced with dextran sulfate sodium. Exp Anim 48: 137-143, 1999.

19. Venkatraman A, Ramakrishna BS, Pulimood AB, Patra S and Murthy S: Increased permeability in dextran sulphate colitis in rats: time course of development and effect of butyrate. Scand J Gastroenterol 35: 1053-1059, 2000.

20. Suzuki A, Hanada T, Mitsuyama K, Yoshida T, Kamizono S, Hoshino T, Kubo M, Yamashita A, Okabe M, Takeda K, Akira S, Matsumoto S, Toyonaga A, Sata M and Yoshimura A: CIS3/ SOCS3/SSI3 plays a negative regulatory role in STAT3 activation and intestinal inflammation. J Exp Med 19: 471-481, 2001.

21. Kajio T, Kawahara K and Kato K: Stabilization of basic fibroblast growth factor with dextran sulfate. FEBS Lett 306: 243-246, 1992.

22. Kjellstrom T and Malmquist J: Effects of heparin and dextran sulphate on the production of collagen and protein in diabetic and non-diabetic human skin fibroblast cultures. Med Biol 61: 186-190, 1983.

23. Bateman JF and Golub SB: Assessment of procollagen processing defects by fibroblasts cultured in the presence of dextran sulphate. Biochem J 267: 573-577, 1990.

24. Morimoto H, Yamashita M, Matsuda A, Ohori M, Miyake H and Fujii T: Characterization of dextran sulfate-induced guinea pig tracheal plasma extravasation. Jpn J Pharmacol 72: 217-221, 1996.

25. Medina C, Videla S, Radomski A, Radomski M, Antolin M, Guarner F, Vilaseca J, Salas A and Malagelada JR: Therapeutic effect of phenantroline in two rat models of inflammatory bowel disease. Scand J Gastroenterol 36: 1314-1319, 2001.

26. Goto M, Kataoka Y, Kimura T, Goto K and Sato H: Decrease of saturation density of cells of hamster cell lines after treatment with dextran sulfate. Exp Cell Res 82: 367-374, 1973.

27. Tessner TG, Cohn SM, Schloemann S and Stenson WF: Prostaglandins prevent decreased epithelial cell proliferation associated with dextran sodium sulfate injury in mice. Gastroenterology 115: 874-882, 1998. 
28. Burg DL, Harrison ML and Geahlen RL: Cell cycle-specific activation of the PTK72 protein-tyrosine kinase in B lymphocytes. J Biol Chem 268: 2304-2306, 1993.

29. Ling KY, Bhalla D and Hollander D: Mechanisms of carrageenan injury of IEC18 small intestinal epithelial cell monolayers. Gastroenterology 95: 1487-1495, 1988.

30. Umemura K, Yanase K, Suzuki M, Okutani K, Yamori T, and Andoh T: Inhibition of DNA topoisomerases I and II, and growth inhibition of human cancer cell lines by a marine microalgal polysaccharide. Biochem Pharmacol 66: 481-487, 2003.

31. Furukawa $\mathrm{K}$ and Bhavanandan VP: Influences of anionic polysaccharides on DNA synthesis in isolated nuclei and by DNA polymerase alpha: correlation of observed effects with properties of the polysaccharides. Biochim Biophys Acta 740: 466-475, 1983.

32. Vetuschi A, Latella G, Sferra R, Caprilli R and Gaudio E: Increased proliferation and apoptosis of colonic epithelial cells in dextran sulfate sodium-induced colitis in rats. Dig Dis Sci 47: 1447-1457, 2002.
33. Shinozaki M, Watanabe T, Kubota Y, Sawada T, Nagawa H and Muto T: High proliferative activity is associated with dysplasia in ulcerative colitis. Dis Colon Rectum 43: S34-S39, 2000.

34. Arai N, Mitomi H, Ohtani Y, Igarashi M, Kakita A and Okayasu I: Enhanced epithelial cell turnover associated with p53 accumulation and high p21WAF1/CIP1 expression in ulcerative colitis. Modern Pathol 12: 604-611, 1999.

35. Tsuchiya T, Imaeda A, Kiho T and Ukai S: Detoxification of paraquat poisoning: effects of carbohydrate sulfate, alkylsulfate and alkylsulfonate on active oxygen. Biol Pharm Bull 18: 1700-1704, 1995

36. Araki Y, Sugihara $\mathrm{H}$ and Hattori T: The free radical scavengers edaravone and tempol suppress experimental dextran sulfate sodium-induced colitis in mice. Int J Mol Med 17: 331-334, 2006. 